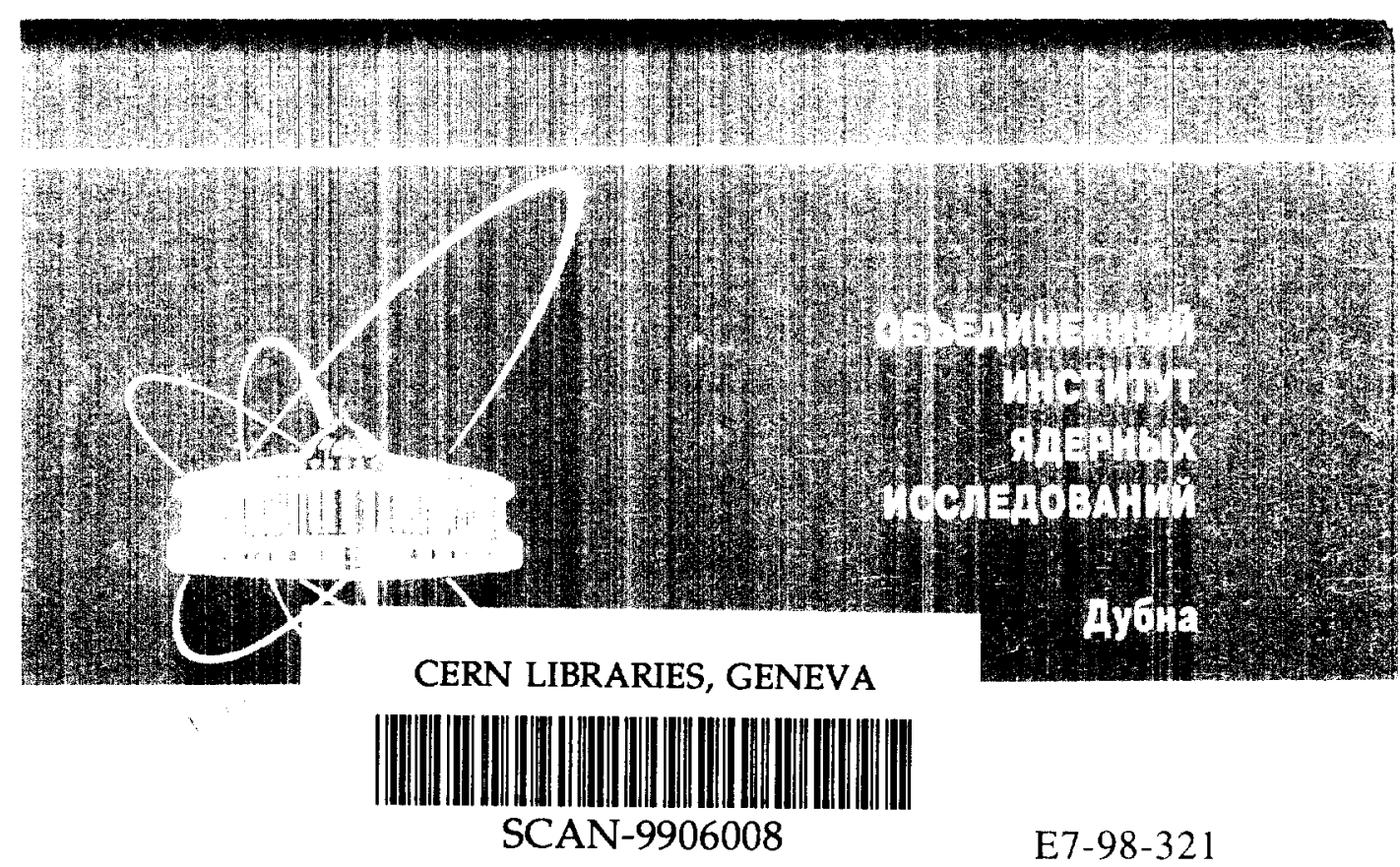

D.V.Vanin*, G.I.Kosenko, G.D.Adeev*

\title{
LANGEVIN CALCULATIONS \\ OF FISSION FRAGMENT MASS DISTRIBUTION \\ IN FISSION OF EXCITED NUCLEI
}

Submitted to «Physical Review C»

*Omsk State University, Department of Theoretical Physics, Mira Prospekt 55-a, Omsk, 644070, Russia 



\section{Introduction}

In recent years fairly extensive experimental material has been accumulated on the observed fission fragment distributions as functions of various parameters of the compound nucleus such as excitation energy, angular momentum and fissility parameter (or parameter $Z^{2} / A$ ). This material was systematized and analyzed in $[1,2,3]$. Analysis of the modern understanding of the formation of the fragment distributions shows $[1,2,3]$ that overall it does not correspond to the bulk of the existing experimental data. The elucidation of the mechanism of formation of the observed fission fragment distribution (mass, energy, angular) on the basis of a systematic dynamical treatment of the process remains one of the principal unresolved problems of fission physics. This situation is due in the first place to the well known [4] difficulties of constructing a dynamical theory of an extremely non-equilibrium process such as fission. Among several theoretical schemes for describing fission dynamics, the stochastic approach $[5,6,7]$ is the most promising and evolving.

In last decade a stochastic approach $[5,6,7]$ based on the FokkerPlank equation (FPE) $[8,9]$ or on the set of stochastic Langevin equations (LE), which is equivalent $[10,11]$ to the multidimensional FPE, has been successfully used to describe many salient features of fusion, deep inelastic heavy ion collision, and induced fission (see recent reviews $[6,7]$ where relevant literature can be found). Until recently the FPE has been used for these studies rather than the LE because the latter contains random force and requires gathering many fission events to reduce statistical error and obtain confident results. Only the impressive development of computers enables us to apply practically $[11,12,13]$ the LE to study nuclear dissipative phenomena. Almost all the problems of collective nuclear dynamics are multidimensional but the multidimensional FPE can be solved only approximately. On the other hand the multidimensional LE can be solved numerically without any approximations. With this approach one can study many interesting properties of induced fission, such as fission fragment energy and mass distributions, dynamical competition between fission and particle emission and so on.

Several papers have already been published $[12,13,14,15,16]$ to 
explain the large variances of the fission-fragment kinetic energy distribution, and rather satisfactory results have been obtained for the parameters of the fission-fragment kinetic energy distribution.

On the contrary there is not even one publication with a theoretical investigation of the main features of the fission- fragment mass distribution using the LE.

In the present paper we report the first results of a calculation of fission-fragment mass distributions performed with the Langevin approach to fission dynamics.

All transport coefficients of the LE were calculated with a macroscopic approach without taking into account the effect of pairing correlations of nucleons and shell effects, since we considered the fission of sufficiently excited compound nuclei (with excitation energy $E^{*} \geq 25 \mathrm{MeV}$ and temperature $T \geq 1 \mathrm{MeV}$ ). The calculations were carried out for a wide range of the fissility parameter $\left(Z^{2} / A\right)$. Two versions of the liquid drop model were used in the calculations: the LDM with the sharp surface of nucleus and the LDM with the diffuse surface [17] with Sierk's parameters [18]. These models predict the different values of the potential energy of the fissioning nucleus at the saddle point as well as at the scission region, the stiffnesses with respect to mass-asymmetry coordinate are also different in both models. Therefore, it is interesting to check the predictions of the dynamical calculations of the fission fragment mass distributions using two different rnass formulae.

\section{Formulation of model}

\subsection{Parametrization of nuclear surface, potential energy, inertia and dissipation}

For parametrization of the nuclear surface we used a two-parametric family of shapes based on Cassini ovaloids which have been introduced in nuclear physics in $[19,20]$.

In cylindrical coordinates the surface of the nucleus is given by [20] 


$$
\rho_{s}^{2}=\sqrt{\frac{1}{c_{v}^{4}}+4 \frac{\varepsilon}{c_{v}^{2}}(z-\kappa|z|)^{2}}-\frac{\varepsilon}{c_{v}^{2}}-z^{2} .
$$

Here $z$ is the coordinate along the symmetry axis, $\rho$ is the one perpendicular to $z, \rho_{s}$ is the value of $\rho$ at the nuclear surface. $\varepsilon$ is the elongation parameter and $\kappa$ is the coordinate of mass asymmetry which defines the mass ratio of the nascent fragments. In the Eq.(1) a scale factor $c_{v}$ ensures volume conservation of the nucleus. In the symmetrical case $(\kappa=0)$ the nuclear shapes corresponding to the Eq.(1) are Cassini ovaloids $[19,21]$, for the case $\kappa>0$ we obtain different asymmetric forms. The condition $\varepsilon<\frac{1}{2}(1+\kappa)^{-2}$ gives axially symmetrical ovaloids, $\frac{1}{2}(1+\kappa)^{-2}<\varepsilon<\frac{1}{2}(1-\kappa)^{-2}$ leads to pear-shaped figures and finally, $\varepsilon>\frac{1}{2}(1-\kappa)^{-2}$ leads to asymmetrical figures with a neck on the surface of the nucleus.

The value $\varepsilon=1$ at any $\kappa$ corresponds to the figures with zero neck radius and can be considered as one (simplest) scission condition. But often it was supposed $[4,22]$ that scission occurs at the critical deformation with relatively thick neck radius.

In [19] it was shown that Cassini ovaloids with an accuracy that is surprising for the single-parameter family of shapes describe the characteristics of the saddle-point shapes (the barrier heights and the effective moments of inertia) obtained by Strutinsky et al. [23] with using the numerical solution of the exact integro-differential equation for equilibrium shape in a static drop model for arbitrary deformation. The situation is worse for the mass-asymmetric saddle-point shapes when for the adequate description it is necessary to introduce a few parameters of asymmetrical shapes up to ten [24].

The deformation parameters $\varepsilon$ and $\kappa$ are treated as collective coordinates $\left(q_{1}, q_{2}\right)=(\varepsilon, \kappa)$.

The potential deformation energy, as mentioned above, is calculated using two versions of the liquid drop model. The LDM with the sharp surface was used with parameters taken from [25] and the finite range LDM [17] was used with parameters taken from [18].

As usually the potential energy is calculated as a sum of the Coulomb and nuclear interaction energies. In the LDM with the sharp surface of 
the nucleus the surface energy plays the role of the nuclear interaction energy. The LDM with the diffuse surface takes into account the finite range of the nuclear forces by means of Yukawa-plus-exponential folding function [17]. The resulting potential energy surfaces for a few fissioning systems as a function of parameters $\varepsilon$ and $\kappa$ are shown in Fig.1 and Fig.2.

The inertia tensor is calculated by means of the Werner-Wheeler approximation for incompressible irrotational flow [26]. As it was shown in Ref.[27] the Werner-Wheeler method rather correctly allows the calculation of the inertia tensor practically for all shapes of the fissioning system. For dissipation we used two different models: two-body hydrodynamical viscosity and one-body wall-and-window dissipation [28, 29]. The following expression was applied to calculate the friction tensor for wall-and-window dissipation:

$$
\begin{gathered}
\gamma_{i j}=\frac{1}{2} \rho_{m} \bar{v}\left\{\frac{\partial R}{\partial q_{i}} \frac{\partial R}{\partial q_{j}} \Delta \sigma+\pi \int_{z_{m i n}}^{z}\left(\frac{\partial \rho_{s}^{2}}{\partial q_{i}}+\frac{\partial \rho_{s}^{2}}{\partial z} \frac{\partial D_{1}}{\partial q_{i}}\right) \times\right. \\
\times\left(\frac{\partial \rho_{1}^{2}}{\partial q_{j}}+\frac{\partial \rho_{s}^{2}}{\partial z} \frac{\partial D_{1}}{\partial q_{j}}\right)\left(\rho_{s}^{2}+\left(\frac{1}{2} \frac{\partial \rho_{i}^{2}}{\partial z}\right)^{2}\right)^{-\frac{1}{2}} d z+\pi \int_{z_{N}}^{z_{\max }}\left(\frac{\partial \rho_{s}^{2}}{\partial q_{i}}+\frac{\partial \rho_{i}^{2}}{\partial z} \frac{\partial D_{2}}{\partial q_{i}}\right) \times \\
\left.\times\left(\frac{\partial \rho_{s}^{2}}{\partial q_{j}}+\frac{\partial \rho_{s}^{2}}{\partial z} \frac{\partial D_{2}}{\partial q_{j}}\right)\left(\rho_{s}^{2}+\left(\frac{1}{2} \frac{\partial \rho_{i}^{2}}{\partial z}\right)^{2}\right)^{-\frac{1}{2}} d z\right\}
\end{gathered}
$$

where $\rho_{m}$ is the mass density of the nucleus, $\bar{v}$ is an average nucleon speed inside the nucleus, $\Delta \sigma$ is an area of the window between two parts of the system, $R$ is the distance between centers of mass of future fragments, $D_{1}, D_{2}$ are positions of centers of mass of the two parts of the fissioning system relative to the center of mass of the whole system. $z_{\min }$ and $z_{\max }$ are the left and right ends of the nuclear shape, $z_{N}$ is the position of the neck plane which divides the nucleus into two parts. For the symmetrical forms this formula is reduced and becomes similar to that which is presented in Ref.[29] where a misprint in the sign before the term with the derivative $\partial D / \partial q_{i}$ has taken place. We used the full wall-and-window dissipation formula, i.e. with coefficient $k_{s}=1$ (see Ref. [30]) before the wall formula; however, the comparison between the calculated and the experimental results of the mean values of the total 


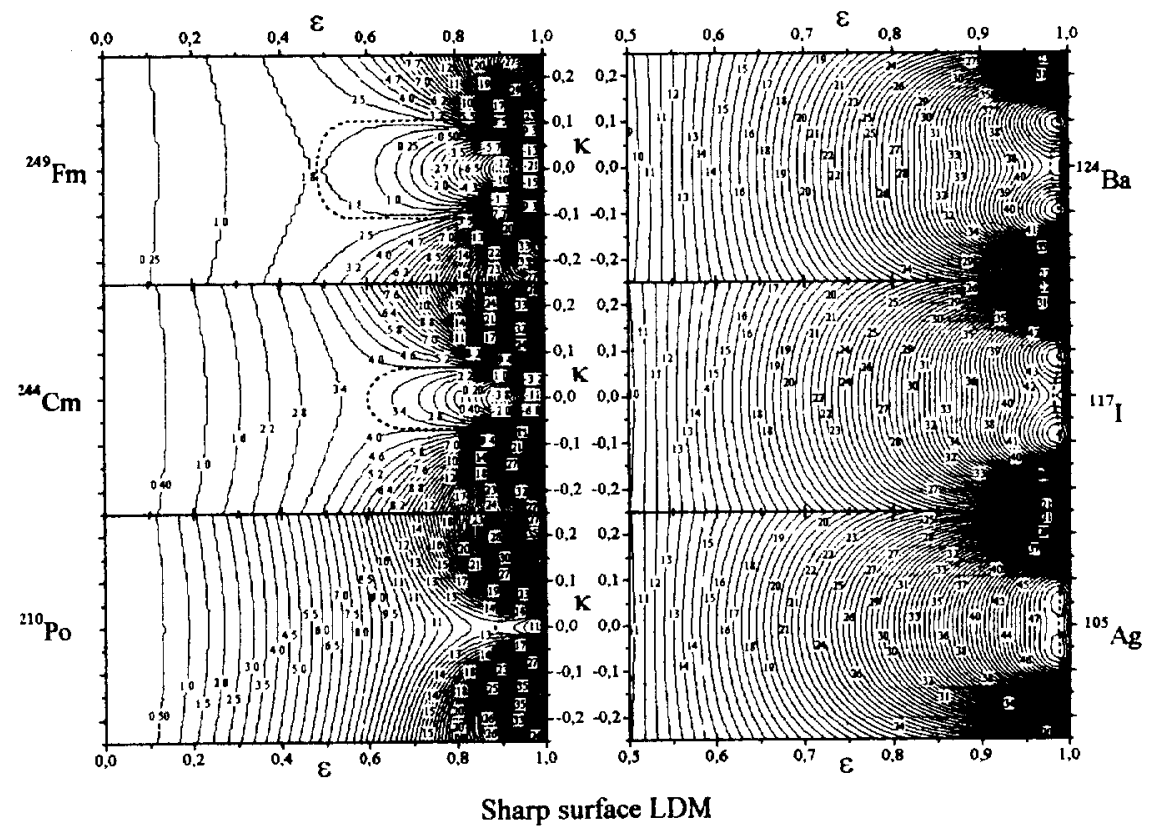

Figure 1: The potential energy surfaces for nuclei ${ }^{249} \mathrm{Fm},{ }^{244} \mathrm{Cm},{ }^{210} \mathrm{Po}$, ${ }^{124} \mathrm{Ba},{ }^{117} \mathrm{I},{ }^{105} \mathrm{Ag}$ calculated within the LDM with the sharp surface of the nucleus. Contour energies are in $\mathrm{MeV}$. 


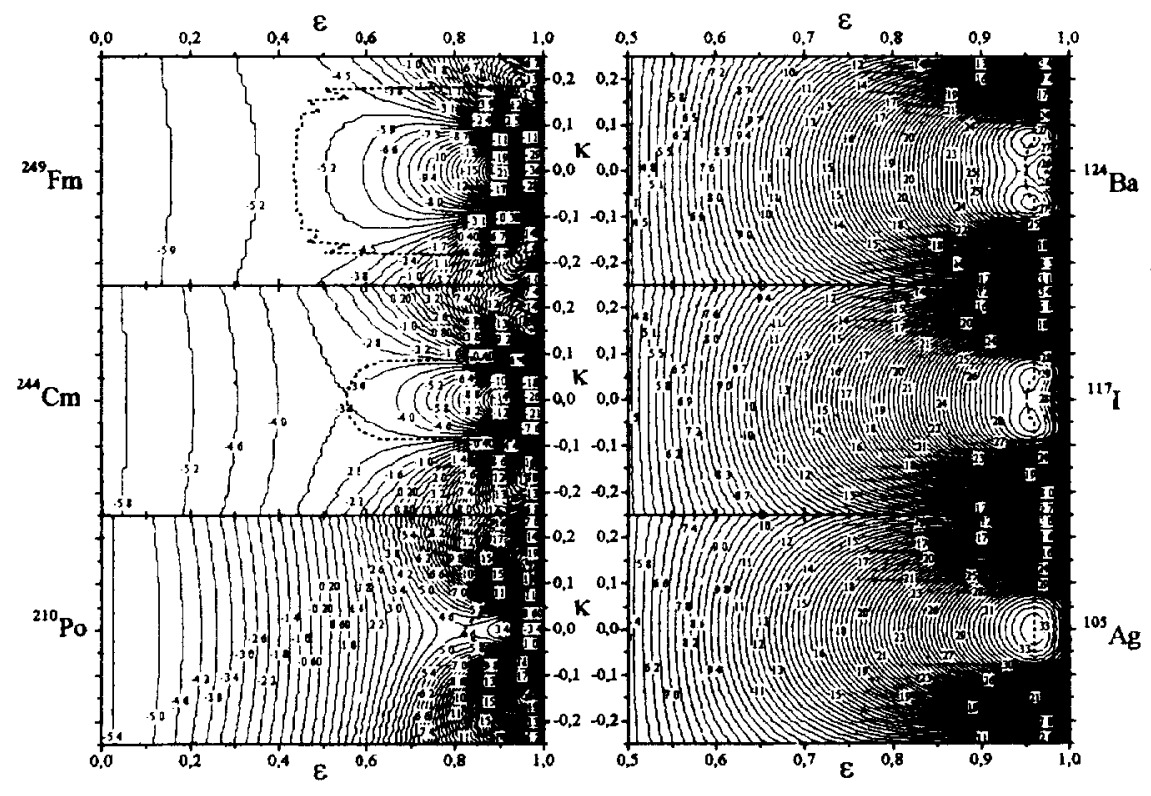

Finite range LDM

Figure 2: The potential energy surfaces for the same nuclei as in Fig.1 calculated within the finite range LDM. 
kinetic energy of the fission fragments for all fissioning nuclei throughout the periodic system suggests [30] the limits $0.2 \leq k_{s} \leq 0.5$. The value of $k_{s}$ could depend upon both the excitation energy and the type of collective motion.

\subsection{Basic equations}

The evolution of collective degrees of freedom is considered in the stochastic approach $[5,6,7]$ as the motion of Brownian particles [31] in a medium (heat bath) formed by the set of single-particle degrees of freedom. The coupled Langevin equations used in the dynamical calculations have the form:

$$
\begin{aligned}
\dot{q}_{i} & =\mu_{i j} p_{j} \\
\dot{p}_{i} & =-\frac{1}{2} p_{j} p_{k} \frac{\partial \mu_{j k}}{\partial q_{i}}-\frac{\partial V}{\partial q_{i}}-\gamma_{i j} \mu_{j k} p_{k}+\theta_{i j} \xi_{j}, \\
i, j, k & =1 \ldots N .
\end{aligned}
$$

Where $\mathbf{q}=(\varepsilon, \kappa)$ are the collective coordinates, $\mathbf{p}=\left(p_{\varepsilon}, p_{\kappa}\right)$ are the momenta which conjugated them, $V(\mathbf{q})$ is the potential energy, $m_{i j}\left(\left\|\mu_{i j}\right\|=\left\|m_{i j}\right\|^{-1}\right)$ is the tensor of inertia, $\gamma_{i j}$ is the friction tensor, and $\theta_{i j} \xi_{j}$ is a random force, $\xi_{j}$ is a random variable satisfying the relations:

$$
\begin{array}{r}
<\xi_{i}>=0,<\xi_{i} \xi_{j}>=2 \delta_{i j}, \\
<\xi_{i}\left(t_{1}\right) \xi_{j}\left(t_{2}\right)>=2 \delta_{i j} \delta\left(t_{1}-t_{2}\right) .
\end{array}
$$

The second equation means that no time-correlated random force is assumed, i.e. Markovian approximation. The amplitudes $\theta_{i j}$ of the random force are related to the diffusion tensor $D_{i j}$ by the equation:

$$
D_{i j}=\theta_{i k} \theta_{k j} \text {. }
$$

The tensor $D_{i j}$ satisfies the Einstein relation 


$$
D_{i j}=T \gamma_{i j}
$$

Here $T$ is the nuclear temperature which is determined by the Fermigas formula $[32,33]$

$$
T=\left(E_{i n t} / a\right)^{1 / 2} .
$$

Where $E_{\text {int }}$ is the internal excitation energy of the nucleus and $a$ is the level density parameter. For the latter we took the value of $a=A / 10$ ( $A$ is the mass number of the fissioning nucleus).

In our calculations we used the energy conservation law in the form

$$
E^{*}=E_{i n t}+E_{k}+V(\mathbf{q})
$$

where $E^{*}$ is the total excitation energy of the nirleus and $E_{k}$ is the kinetic energy of the collective degrees of freedorn.

The initial conditions for the dynamical calculations were chosen by Neumanr method (sometimes the so-called hit and miss method i with the generating function

$$
f \sim \exp \left(-\frac{V(\mathbf{q})+E_{i}}{T}\right),
$$

that is we used the Maxwell-Boltzmann distribution function. The $\mathbf{q}$ values were found at the ridge line separating the ground state from the fission valley. Initially the momentum $p_{s}$ was always directed toward the scission region. This choice of initial conditions is fully consistent with ideas that form the conceptual framework of the statistical model [33] and can be considered as the generalization of the initial conditions at the saddle point which are commonly used in the dynamical LDM calculations $[34,35,36]$.

\section{Results and discussions}

As mentioned above, the potential energy surfaces are shown in Fig.1 and Fig.2. The surfaces are divided into two groups in the same manner as in Refs.[3, 37, 38]. Light nuclei with the parameter $Z^{2} / A<32$ have 
saddle points very close to scission configurations; therefore the mass distributions for these nuclei are defined mainly at the saddle point. Whereas heavy nuclei with the parameter $Z^{2} / A>32$ have a sufficiently long descent from the saddle point to scission, and their mass distributions are influenced by the descent dynamics. It can be seen that the position of the ridge line (thick dashed line) which separates the ground state region and the scission one is cardinally different for light $\left({ }^{105} \mathrm{Ag}\right.$, $\left.{ }^{117} \mathrm{I},{ }^{124} \mathrm{Ba}\right)$ and heavy $\left({ }^{210} \mathrm{Po},{ }^{244} \mathrm{Cm},{ }^{249} \mathrm{Fm}\right)$ nuclei. Moreover, the potential energy surfaces of light nuclei are significantly different for the LDM with the sharp surface of nucleus and for the LDM with the diffuse surface. For the LDM with the sharp nucleus surface, the ridge line is closer to scission than for the finite range LDM. And throughout the whole range of the calculated nuclei, the difference of the potential energy between the saddle point and scission in the LDM with the diffuse surface is greater than that in the LDM with the sharp surface. It should be stressed that the most obvious difference in landscapes of the potential energy surfaces of the two versions of the LDM is observed at the scission region (see, for example, the potential energy for ${ }^{105} \mathrm{Ag}$ in the finite range LDM there is only one peak at the scission region with a maximum for symmetrical shapes at $\kappa=0$ whereas in I.DM [25] there are two peaks at the scission region with a minimum for symmetrical shapes). Also fission barriers calculated with the finite surface diffuseness I.DM are lower than fission barriers of the sharp surface LDM, especially for light nuclei as was shown in Refs.[17, 18].

Results of our dynamical calculations are presented in Figs.3-6 for one-body dissipation only, because in several calculations $[6,12,16$, 39] of the prescission neutron multiplicities and fragment kinetic energy it was shown that the one-body type of nuclear viscosity is preferable for the consistent description of the data. About $10^{4}$ trajectories were calculated for each nucleus and for each excitation energy. Our detailed calculations with both types of nuclear viscosity will be discussed in further publications.

The calculated mass distributions in Fig. 3 and Fig. 4 reflect the features of the potential energy surfaces which are presented for the same systems in Fig.1 and Fig.2. The presented results of our calculations 


\section{Sharp surface LDM}
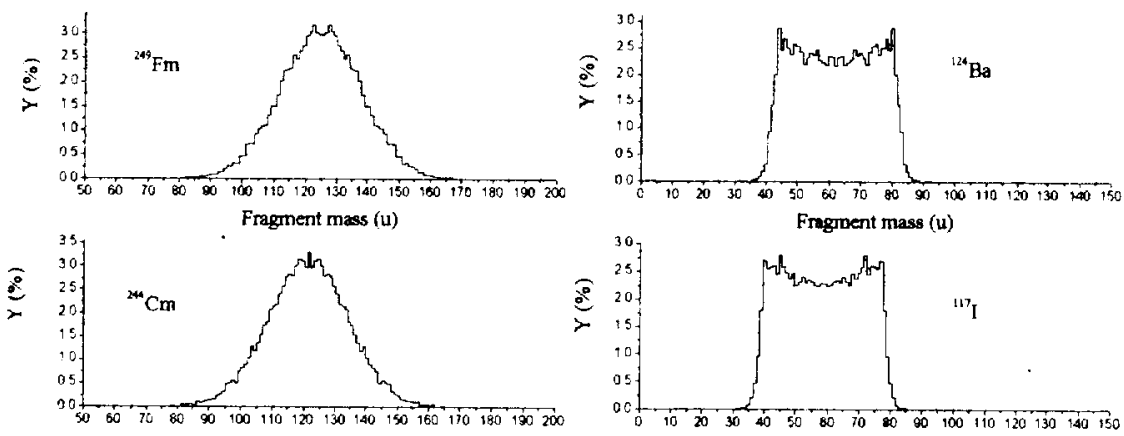

Fragment mass (u) Fragment mass (u)
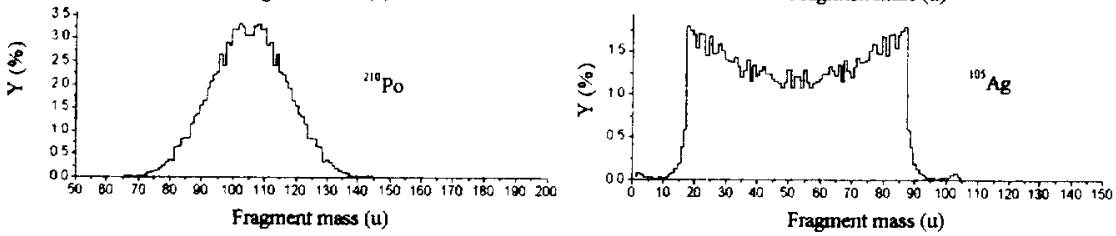

Figure 3: The mass distributions of fission fragments of ${ }^{249} \mathrm{Fm},{ }^{244} \mathrm{Cm}$, ${ }^{210} \mathrm{Po},{ }^{124} \mathrm{Ba},{ }^{117} \mathrm{I},{ }^{105} \mathrm{Ag}$ compound systems calculated within the LDM with the sharp surface of nucleus. 


\section{Finite range LDM}
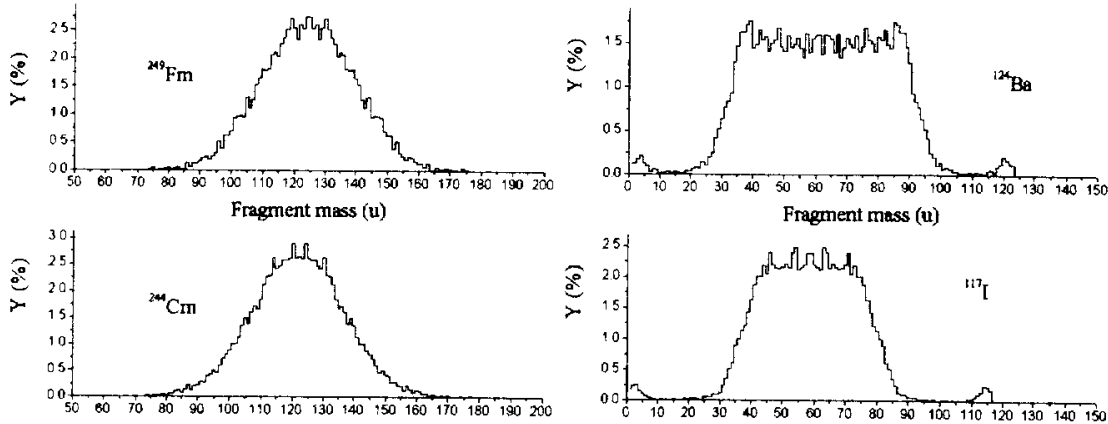

Fragment mass (u)

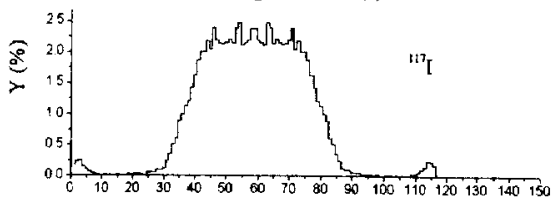

Fragment mass (u)
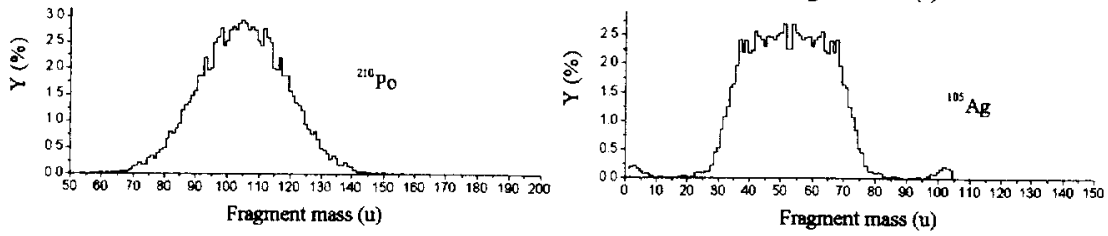

Figure 4: The same as Fig.3, but calculated within the finite range LDM. 
are normalized by a full number of fission fragments (so-called $200 \%$ normalization). It is well known that one very interesting and salient prediction of the static calculations $[40,41]$ in the LDM or in another macroscopic model $[42,43]$ is the existence of the so-called BusinaroGallone (BG) point as a function of the fissility parameter (or of the parameter $Z^{2} / A$ ) in which the nucleus completely loses its stability to mass-asymmetric deformations. Different versions of the LDM predict the different values of $Z^{2} / A$ for the BG point. The LDM with the sharp surface gives $\left(Z^{2} / A\right)_{B G} \simeq 19$, whereas the LDM with the diffuse surface gives $\left(Z^{2} / A\right)_{B G} \simeq 22 \div 23$ in dependence on the set of parameters $[17,18]$.

The fission fragment mass distributions would be gaussian-like shapes for nuclei with $Z^{2} / A>\left(Z^{2} / A\right)_{B G}$. In close vicinity of the $\mathrm{BG}$ point, shape broadening of the mass distribution should take place. Strictly speaking, for nuclei with $Z^{2} / A=\left(Z^{2} / A\right)_{B G}$ the mass distributions should be flat in the wide range of mass near $A_{C N} / 2$, and for lighter nuclei the fission fragment mass distribution should have an $U$-like shape $[3,37,41]$. Such transformation of the shape of the mass distributions is actually observed experimentally. It is interesting to see how the theory can describe this transformation of the mass distribution as a function of the parameter $Z^{2} / A$ of the compound nucleus.

It is shown in Fig.3 and Fig.4 that qualitatively we correctly reproduced this transition from a gaussian-like distribution (for $Z^{2} / A>$ $\left.\left(Z^{2} / A\right)_{B G}\right)$ to $\mathrm{U}$-like distributions (for $Z^{2} / A<\left(Z^{2} / A\right)_{B G}$ ), but the position of the $B G$ point in our calculation lies at $Z^{2} / A \approx 25$. However, for a more accurate determination of the $B G$ point it is necessary to carry out calculations for a larger number of nuclei.

In Fig. 5 and Fig. 6 the calculated values of the variances of the fissionfragment mass distributions are compared with experimental data as a function of the parameter $Z^{2} / A$ and of the temperature of the compound nucleus.

The variances $\sigma_{M}^{2}$ as a function of the parameter $Z^{2} / A$ together with the experimental data are presented in Fig.5. In this figure it is shown that we have fairly good quantitative agreement with data for the interval $25<Z^{2} / A<35$. It should be noted that calculated values of $\sigma_{M}^{2}$ for 


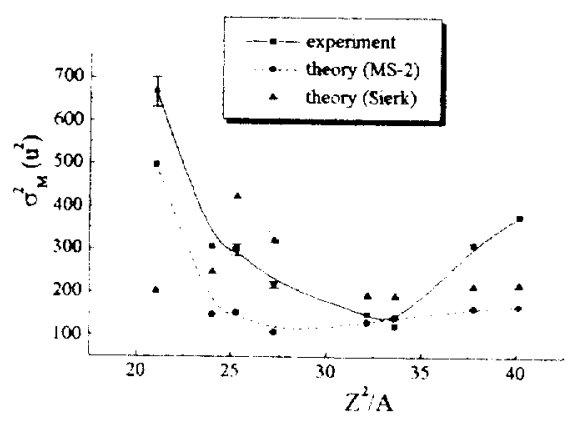

Figure 5: The variances of the mass distributions of the fission fragments versus the parameter $Z^{2} / A$. The squares and the solid line are the reduced to $L=0$ experimental data taken from [38]. Theoretical results are shown by the circles and the dashed line for the LDM with the sharp surface of the nucleus and the upper triangles and the dotted line for the finite range. LDM.

these nuclei are significantly larger than the values of $\sigma_{M}^{2}$ predicted by statistical model [8] (about factor 2). When the descent from ridge to scission takes place extremely slowly, the fissioning system can "forget" its state at prior times. If the descent occurs during a finite time (with finite velocity) the fissioning system preserves "memory" of the large values of $\sigma_{M}^{2}$ (because of the smaller stiffness of the potential energy respective mass asymmetry deformations at earlier times). Moreover, the faster the descent of the nucleus from the ridge to scission. the larger the "remembered" values of the variance of the mass-asymmetry coordinate will be. Such an interpretation of the "memory" of the fissioning system of its prehistory was discussed in detail at a quantitative level in [8]. Therefore the calculated values of $\sigma_{M}^{2}$ will be dependent strongly on the velocity of the descent from the ridge to scission and finally on the magnitude of the nuclear viscosity. Wada and $A$ be [44] have arrived at a similar conclusion about the consequences of the dynamical evolution from the saddle to scission, especially for heavy fissioning systems.

As it is seen from Fig.5 our calculations do not reproduce the observed experimental growth of variance for nuclei with $Z^{2} / A>35$. From the discussion above it is possible to make the assumption that the dissipation used by us in the calculation with $k_{s}=1$, which corresponds to highly overdamped collective motion is too large for a description of the fission-fragment mass distributions of hot compound nuclei. Also our calculations do not reproduce the growth of variances $\sigma_{M}^{2}$ for light fissioning nuclei with $Z^{2} / A<25$. For these nuclei it is necessary to con- 


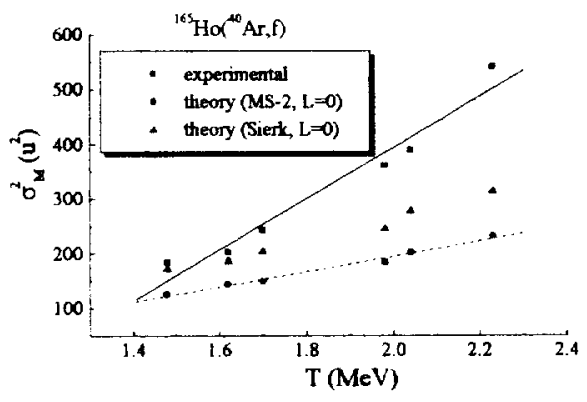

Figure 6: The variances of mass distributions of fission fragments for the compound nucleus ${ }^{205}$ At formed in reaction ${ }^{165} \mathrm{Ho}\left({ }^{40} \mathrm{Ar}, f\right)$ at the range of projectile energy: $E_{\text {lab }}=$ $180,192,200,230,243,280 \mathrm{MeV}$ are drawn as a function of the saddle point temperature of the compound nucleus. Squares are the experimental points taken from [38]. Theoretical results are shown by circles and upper triangles for the LDM with the sharp surface of the nucleus and for the finite range LDM, respectively. All data are interpolated by solid, dashed and dotted straight lines.

sider their dynamical evolution not from the ridge but from the ground state. The results of the calculation of the fission fragment mass distributions and their variances preformed within the finite range LDM show that they qualitatively similar to those obtained within the sharp surface LDM for heavy nuclei. But there are significant quantitative differences in the variances of the mass distributions for light nuclei.

It can be seen from Fig. 6 that the saddle point temperature dependence $\sigma_{M}^{2}(T)$ in reaction ${ }^{165} H o\left({ }^{40} A r, f\right)$ at six projectile energies $\left(E_{l a b}=180,192,200,230,243,280\right.$

$\mathrm{MEV}$ ) calculated in the present work without allowance for quantum fluctuations is practically a straight line. The slope of the calculated dependence of $\sigma_{M}^{2}(T)$ is significantly smaller than the slope of the experimental one. The explanation of this large difference is the following. The theoretical dependencies of $\sigma_{M}^{2}(T)$ are calculated for zero angular momentum of the compound nucleus, whereas the experimental dependencies of $\sigma_{M}^{2}(T)$ correspond to all possible values of the angular momentum realized in the reaction in question. It is known [38] that a substantial increase of the angular momentum of the compound nucleus leads to a broadening of the fission fragment mass distributions. The 
extension of our theoretical scheme for rotating compound nuclei will improve the agreement between the experimental data and theoretical results.

\section{Summary and outlook}

We have applied the two-dimensional stochastic approach to fission dynamics based on Langevin equations to study the main features of the fission fragment mass distribution of excited fissioning nuclei.

Using two versions of the LDM and the one-body mechanism of nuclear viscosity we have performed Langevin Monte-Carlo calculations of the fission fragment mass distribution for fissioning systems in the wide range of parameter $Z^{2} / A$ and of excitation energy $E^{*}$. The calculated mass distributions $Y(M)$ and their variances are found to be in satisfactory qualitative agreement with experimental data and with results of previous calculations [8] performed with using multidimensional FPE.

Of course, the model used in our calculations needs further refinements. First, it is clearly necessary to replace the initial conditions given by Eq.(9) with more natural initial conditions at the ground state. It is especially important for the light fissioning nuclei for which the ridge is close to the scission region. Second, it is necessary to incorporate into the Langevin scheme the evaporation of the light particles (so-called prescission particles) and to study the competition between particle emission and fission dynamically $[6,7,16]$. Third, in the LE, for hot nuclei the free energy must replace the potential energy [5]. In the calculations of the free energy of the fissioning system one has to take into account the dependence of the level density parameter on deformation. Lastly, it is desirable to develop the three-dimensional Langevin approach with inclusion of the additional collective coordinate (constriction parameter) for the simultaneous calculation of the mass-energy distribution of fission fragments. Studies in these directions are now in the progress. 


\section{Acknowledgments}

We are grateful to Drs. A.Ya.Rusanov, V.S.Salamatin and H.J.Krappe for stimulating and useful discussions and to Prof. M.G.Itkis for the permanent support of this study.

This work was supported in part by International Association of Cooperation with Scientists from the Independent States of the Former Soviet Union (grant no. INTAS-93-1560).

\section{References}

[1] Yu.Ts.Oganessian and Yu.A.Lazarev, "Heavy ions and nuclear fission.", in: Treatise on Heavy Ion Science, Vol.4, edited by D.A.Bromley (Plenum 1995, New York, 1985), p.1

[2] Nuclear Fission Process, edited by C.Wagemans (CRC Press, Boca Raton, USA, 1991)

[3] M.G.Itkis and A.Ya.Rusanov, Russian J.Part.Nucl. 29, 389 (1998)

[4] M.Brack, J.Damgaard, A.S.Jensen, H.C.Pauli, V.M.Strutinsky, and C.Y.Wong, Rev.Mod.Phys. 44, 320 (1972)

[5] H.J.Krappe, Proc. Int. Workshop on Dynamical Aspects of Nuclear Fission, Dubna, JINR, 1992, p.51

[6] Y.Abe, S.Ayik, P-G.Reinhard, and E.Suraud, Phys.Rep. 275, 49 (1996)

[7] P.Fröbrich and I.I.Gontchar, Phys.Rep. 292, 131 (1998)

[8] G.D.Adeev and V.V.Pashkevich, Nucl.Phys. A502, 405c (1989); G.D.Adeev, I.I.Gontchar, V.V.Pashkevich, N.I.Pischasov, and O.I.Serduk, Sov. J. Part. Nucl. 19, 529 (1988)

[9] F.Scheuter, C.Grégoire, H.Hofmann, and J.R.Nix, Phys.Lett 149B, 303 (1984)

[10] S.Chandrasekhar, Rev. Mod. Phys. 15, 1 (1943)

[11] Y.Abe, S.Grégoire, and H.Delagrange, J. de Physique 47, C4-329 (1986); P.Fröbrich and S.Y.Xu, Nucl.Phys. A477, 143 (1988) 
[12] T.Wada, N.Carjan, and Y.Abe, Nucl.Phys. A538, 283c (1992)

[13] G.-R.Tillack, R.Reif, A.Schülke, P.Fröbrich, H.J.Krappe, and H.G.Reusch, Phys.Lett. B296, 296 (1992)

[14] I.I.Gontchar, G.I.Kosenko, N.I.Pischasov, and O.I.Serdyuk, Yad.Fiz. 55, 820 (1992)

[15] J.Bao, Y.Zhuo, and X.Wu, Z.Phys. A 352, 321 (1995)

[16] G.I.Kosenko, I.G.Cagliari, and G.D.Adeev, Yad.Fiz. 60, 404 (1997)

[17] H.J.Krappe, J.R.Nix, and A.J.Sierk, Phys.Rev. C20, 992 (1979)

[18] A.J.Sierk, Phys.Rev. C33, 2039 (1986)

[19] V.S.Stavinsky, N.S.Rabotnov, and A.A.Seregin, Yad.Fiz 7, 1051 (1968); V.V.Pashkevich, Nucl.Phys. A169, 275 (1971)

[20] G.D.Adeev and P.A.Cherdantsev, Phys.Lett. 39B, 485 (1972); G.D.Adeev and P.A.Cherdantsev, Yad.Fiz. 18, 741 (1973)

[21] R.W.Hasse and W.D. Myers, Geometrical Relationships of Macroscopic Nuclear Physics (Springer-Verlag, Berlin, 1988)

[22] U.Brosa, S.Grossmann, and A.Müller, Phys.Rep. 197, 167 (1990)

[23] V.M.Strutinsky, N.Ya.Lyashchenko, and N.A.Popov, Nucl.Phys 46 , $639(1962)$

[24] K.T.R.Davies and A.J.Sierk, Phys.Rev. C 31, 915 (1985)

[25] W.D.Myers and W.J.Swiatecki, Ark.Fys. 36, 343 (1967)

[26] K.T.R.Davies, A.J.Sierk, and J.R.Nix, Phys.Rev. C13, 2385 (1976)

[27] A.A.Seregin, Yad.Fiz. 55, 2639 (1992)

[28] J.Blocki, Y.Boneh, J.R.Nix, J.Randrup, M.Robel, A.J.Sierk, and W.J.Swiatecki, Ann.Phys.(N.Y.) 113, 330 (1978)

[29] A.J.Sierk and J.R.Nix, Phys.Rev. C21, 982 (1980) 
[30] J.R.Nix and A.J.Sierk, in: International Seminar School on HeavyIon Physics, D7-87-68 (Dubna, 1987), p.453; J.R.Nix and A.J.Sierk, Preprint LA-UR-87-133, Los Alamos (1987)

[31] H.A.Kramers, Physica 7, 284 (1940)

[32] A.V.Ignatyuk, International Atomic Energy Report INDC (CCP)223/L (1985); Statistical properties of excited nuclei (Energoatomizdat, Moscow, 1983) [in Russian]

[33] R.G.Stokstad, Treatise on Heavy Ion Science, Bromley D.A., Ed. New York, Plenum 1995, vol.3, p.83

[34] J.R.Nix and W.J.Swiatecki, Nucl.Phys. 71, 1 (1965)

[35] J.R.Nix, Nucl.Phys. A 130, 241 (1969)

[36] R.W.Hasse, Nucl.Phys. A 128, 609 (1968)

[37] M.G.Itkis, Yu.A.Muzychka, Yu.Ts.Oganessian, V.N.Okolovich, V.V.Pashkevich, A.Ya.Rusanov, V.S.Salamatin, G.N.Smirenkin, and G.G.Chubarian, Yad.Fiz. 58, 2140 (1995)

[38] A.Ya.Rusanov, M.G.Itkis, and V.N.Okolovich, Yad.Fiz. 60, 773 (1997)

[39] T.Wada, Y.Abe, and N.Carjan, Phys.Rev.Lett. 70, 3538 (1993)

[40] S.Cohen and W.J.Swiatecki, Ann.Phys.(N.Y.) 22, 406 (1963)

[41] L.G.Moretto, Nucl.Phys A247, 211 (1975)

[42] W.D.Myers, Droplet Model of Atomic Nuclei, IFI Plenum Data Co., New York, NY (1977)

[43] R.W.Hasse, Ann.Phys.(NY) 69, 372 (1971)

[44] T.Wada, Proc. 2nd Tours Symp. on Nuclear Physics, Tours September, 1994; Y.Abe, Proc. 3nd IN2P3-RIKEN Symp. on Heavy Ion Collisions, Tokyo, October, 1994

\section{Received by Publishing Department}

on November 16, 1998. 


\section{The Publishing Department \\ of the Joint Institute for Nuclear Research \\ offers you to acquire the following books:}

Index Title

94-55 Proceedings of the International Bogoliubov Memorial Meeting. Dubna. 1993 (216 p. in Russian and English)

D3,14-95-323 VII School on Neutron Physics. Lectures. Vol.1. Dubna, 1995 (356 p. in Russian and English)

E10,11-95-387 Proceedings of the ESONE International Conference RTD'94 on REAL TIME DATA 1994 with Emphasis on Distributed Front-End Processing. Dubna, 1994 (358 p. in English)

D15-96-18 Proceedings of the International Workshop Charge and Nucleon Radii of Exotic Nuclei. Poznan, 1995 (172 p. in Russian and English)

E9-96-21 Proceedings of Vii ICFA Beam Dynamics Workshop on «Bcam Issues for Multibunch, High Luminosity Circular Colliders». Dubna, 1995 (198 p. in English)

E2-96-100 Proceedings of the 3rd International Symposium «Dubna Deuteron-95». Dubna, 1995 (374 p. in English)

E2-96-224 Proceedings of the VII International Conference «Symmetry Methods in Physics». Dubna, 1996 (2 volumes, 630 p., in English)

E-96-321 Proceedings of the International Conference "Path Integrals: Dubna'96". Dubna, 1996 (392 p. in English)

E3-96-336 Proceedings of the IV International Seminar on Interaction of Neutrons with Nuclei. Dubna, 1996 (396 p. in English)

E3-96-369 Proceedings of the X International Conference «Problems of Quantum Field Theory». Dubna, 1996 (437 p. in English)

E3-96-507 Proceedings of the International Workshop «Polarized Neutrons for Condensed Matter Investigations». Dubna, 1996 (154 p. in English)

D1,2-97-6 Proceedings of the International Workshop «Relativistic Nuclear Physics: from $\mathrm{MeV}$ to $\mathrm{TeV}$ ». Dubna, 1996 (2 volumes $418 \mathrm{p}$. and $412 \mathrm{p}$. in English and Russian)

E7-97-49 Proceedings of the 3rd International Conferense «Dynamical Aspects of Nuclear Fission». Slovakia, 1996 (426 p. in English)

E1,2-97-79 Proceedings of the XIII International Seminar on High Energy Physics Problems. Relativistic Nuclear Physics and Quantun Chromodynamics. Dubna, 1996 (2 volumes, 364 p. and 370 p. in English)

D5,11-97-112 Proceedings of the 9th International Conference aComputational Modelling and Computing in Physics». Dubna, 1996 (378 p. in English) 
E2-97-213 Proceedings of the V International Seminar on Interaction of Neutron with Nuclei "Neutron Spectroscopy, Nuclear Structure, Related Topics». Dubna, 1997 (446 p. in English)

E2,4-97-263 Proceedings of the Third International Conference «Renormalization Group'96». Dubna, 1996 (436 p. in English)

E10-97-272 Proceedings of the Data Acquisition Systems of Neutron Experimental Facilities (DANEF 97). Dubna, 1997 (325 p. in English)

D19-97-284 Proceedings of the International Symposium «Problems of Biochemistry. Radiation and Space Biology". Dubna, 1997 (2 volumes 284 p. and 405 p. in Russian and English)

E2-97-413 Proceedings of the VII Workshop on High Energy Spin Physics - (SPIN'97). Dubna, 1997 (398 p. in English)

Please apply to the Publishing Department of the Joint Institute for Nuclear Research for extra information. Our address is:

\author{
Publishing Department \\ Joint Institute for Nuclear Research \\ Dubna, Moscow Region \\ 141980 Russia \\ E-mail: publish@pds.jinr.dubna.su.
}


Ванин Д.В., Косенко Г.К., Адеев Г.Д.

Ланжевеновские расчеты массовых распределений осколков деления возбужденных ядер

Для изучения свойств массовых распределений осколков деления возбужденных ядер применяется стохастический подход, основанный на уравнениях ЛІанжевена. Получены формы и дисперсии массовых распределений для параметра делимости $20<Z^{2} / A<40$. Результаты расчета сравниваются с данными эксперимента.

Расчет выполнен с использованием двух разновидностей модели жидкой капли. Это модели жидкой капли с предлоложением о резком крае ядра и с пренпононием о диффузном крае ядра.

Работа выполнена в Лаборатории ядерных реакций им. Г.Н.Флерова ОИЯи.

Vanin D.V., Kosenko G.I., Adeev G.D.

E7-98-321

Langevin Calculations of Fission Fragment Mass Distribution in Fission of Excited Nuclei

A stochastic approach based on two-dimensional Langevin equations was applied to study the main features of fission fragment mass distibutions of excited fissioning systems. The forms and the variances of fission fragment mass distributions in the fissility parameter range $20<Z^{2} / A<40$ have been calculated. The calculated fission fragment mass distributions and their variances on the whole are consistent with experimental data.

The calculatios were carried out within two liquid drop models (LDM): the LDM with the sharp surface of the nucleus and the LDM with the finite range of nuclear interaction.

The investigation has been performed at the Flerov Laboratory of Nuclear Reactions, JINR. 


\section{Макет Т.E.Попеко}

Подписано в печать 07.12 .98

Формат $60 \times 90 / 16$. Офсетная печать. Уч.-изд. листов 1,73

Тираж 305. Заказ 51041. Цена 2 р.

Издательский отдел Объединенного института ядериых исследований Дубиа Московской области 